\title{
Authors' Reply to Michael Auerbach's Comment on:“Frequency and Associated Costs of Anaphylaxis- and Hypersensitivity-Related Adverse Events for Intravenous Iron Products in the USA: An Analysis Using the US Food and Drug Administration Adverse Event Reporting System"
}

\author{
Henry Trumbo ${ }^{1}\left[\right.$ Karolina Kaluza $^{2} \cdot$ Syed Numan $^{2} \cdot$ Lawrence T. Goodnough $^{3}$
}

Accepted: 15 February 2021 / Published online: 9 April 2021

(c) The Author(s) 2021

\section{Dear Editor,}

In acknowledgement of comments on our recent report of a comparative analysis of intravenous (IV) iron products using the US Food and Drug Administration (FDA) Adverse Event Reporting System (FAERS) [1], the authors wish to highlight that they respectfully disagree with the final concluding statement in Dr. Auerbach's commentary, which claims that the manuscript is harmful to the field [2]. We would also like to highlight Dr. Auerbach's inaccurate representation of referenced material pertaining to the Wysowski et al. article [3].

Dr. Auerbach's concluding claim that our manuscript is harmful to the field appears based on his opinion that our findings are "inconsistent with the overwhelming preponderance of prospective, randomized published evidence". However, it is well known that randomized controlled trials, which regulatory agencies rely on to approve new medicines, are insufficient on their own for comprehensive conclusions on drug safety because serious and/or rare adverse events may subsequently emerge in post-marketing surveillance programs following large-scale drug exposure. Downing et al. in 2017, writing in JAMA, concluded that "among 222 novel therapeutics approved by the FDA from 2001 through

This reply refers to the comment available at Reply to the commentary https://doi.org/10.1007/s40264-021-01058-y.

Henry Trumbo

htrumbo@stmaryhealthcare.org

1 Pharmacy Department, St. Mary Medical Center, 1201

Langhorne-Newtown Road, Langhorne, PA 19047, USA

2 American Regent, Norristown, PA, USA

3 Stanford University, Stanford, CA, USA
2010, 32\% were affected by a post market safety event" [4] (safety events were defined in this study as withdrawals due to safety concerns, FDA issuance of incremental boxed warnings added in the post-market period, and FDA issuance of safety communications). Therefore, it is entirely possible that large real-world studies may detect safety issues not previously identified by randomized controlled trials. For these reasons, we consider our manuscript to be a valid contribution to the field of iron deficiency anemia treatment [1].

Dr. Auerbach stated in his letter that we "used a methodology specifically proscribed by the US FDA", referencing Wysowski et al. [3]. He then stated in his letter "the authors of which are all from the FDA and concluded not only that 'allergic reactions are possible with all four parenteral iron products [Dr Auerbach's direct quotation from Wysowski et al.]' but also that the current system of spontaneous adverse event reporting, particularly in the absence of head to-head trials, does not allow the relative rates of serious adverse events (SAEs) to be determined [Dr Auerbach's interpretation of the Wysowski et al. conclusion]". While we agree with the quoted statement from Wysowski et al. that "allergic reactions are possible with all four parenteral iron products", as we report similar findings in our study, we would disagree with Dr Auerbach's interpretation of the remainder of the Wysowski et al. conclusion. The Wysowski et al. conclusion actually reads:

"The data presented herein show that allergic reactions are possible with all four parenteral iron products, and it is difficult to determine which product has the largest risk based on sales data, voluntarily submitted adverse event reports, death certificates, ED visits, and observational studies performed to date. Long-term safety studies have not been done. To help differentiate risk among the parenteral iron products, the brand name 
of the product always should be provided on medical records, death certificates, and adverse drug reaction reports (submitted via http://www.fda.gov/medwatch or 1-800-FDA-1088).” [3]

Specifically, Wysowski et al. do not comment on the need for head-to-head trials, but rather the need for long-term safety studies with IV iron preparations (reference 31 in Wysowski et al. refers to investigating associated oxidative stress, endothelial damage, and renal injury). As we argue above, and supported by Downing et al. [4], head-to-head or other randomized controlled trial evidence on its own is insufficient for comprehensive conclusions on drug safety.

While Wysowski et al. acknowledge limitations of all the methods they tested, and, with respect to FAERS, state "Because of underreporting, possible differential product reporting, lack of iron dextran brand names, and incomplete use (denominator) data, incidence rates and relative risk estimates cannot be calculated", nowhere in their article do they suggest that use of the FAERS database is proscribed by the FDA. In fact, in their concluding statement, they recommend to always include brand names when submitting adverse drug reaction reports to the FDA, showing that they viewed the FAERS database to have an ongoing role in signal detection. In addition, while Wysowski and her co-authors were employees of the FDA at the time of writing of the article, the article contains a disclaimer: "The views expressed are those of the authors and do not necessarily represent the official position of the U.S. Food and Drug Administration". Thus, Dr. Auerbach's statement that the methodology is proscribed by the FDA is not an accurate representation of the Wysowski et al. conclusions.

In our manuscript, we used disproportionality analysis of spontaneous reports to the FAERS with an extensive range of sensitivity analyses to minimize potential bias, and we were careful to comment solely on reporting rates, avoiding any claim of absolute incidence rate differences or relative risk estimates [1]. Disproportionality analysis is an established method for signal detection in post-marketing pharmacovigilance research, and is an essential component of the process used by the European Union's Pharmacovigilance Risk Assessment Committee for detecting, validating, and confirming adverse drug reactions [5].

Thus, our manuscript used internationally accepted methods and, therefore, represents a valid contribution to the field of iron-deficiency anemia treatment [1].

Regarding the other specific points raised by Dr. Auerbach, we respectfully provide the following rebuttals:

1. The lack of differences in hypersensitivity and anaphylactic reactions in the FIRM trial between ferumoxytol and ferric carboxymaltose (reference 12 in our publication) was not unexpected due to the rarity of these events in the randomized trial context and the fact that this trial excluded patients with a prior history of allergies to drugs that did not include those under investigation. As we have argued, randomized controlled trial evidence on its own is insufficient for comprehensive conclusions on drug safety.

2. In response to the criticism that we did not mention all randomized controlled trial evidence in our discussion, we do not consider this to be a valid criticism of our article for the following reasons: first, the aim of this study was not to provide a systematic review and meta-analysis of available clinical trial data; second, randomized controlled trial evidence on its own is insufficient for comprehensive conclusions on drug safety; third, we specifically included the example of the FIRM trial in our discussion because its primary endpoint was safety and it illustrated the important issue of exclusion criteria that bias against the likelihood of allergic reactions occurring (a feature common to many randomized controlled trials of IV iron preparations); fourth, we explained in our introduction that insufficient data were available in FAERS on adverse reactions with ferric derisomaltose (iron isomaltoside) due to its recent US approval date, so discussion of this preparation was not relevant to our publication.

3. We did not discuss hypophosphatemia because the focus of the current article was necessarily on hypersensitivity and anaphylactic reactions. Thus, we do not consider this to be a valid criticism.

4. While we potentially could have included the Achebe and DeLoughery meta-analysis [6] in our discussion, this does not change our central argument that the small sample sizes and selection criteria of randomized controlled trials do not permit, on their own, an adequate evaluation of hypersensitivity and anaphylactic reactions with IV iron formulations.

5. While we would agree that failing to distinguish highmolecular weight and low-molecular weight dextran was a major limitation of the Wang et al. study [7], we considered the use of the reference to be valid when considering the historical data concerning the association of IV iron preparations with hypersensitivity and anaphylactic reactions. Indeed, we did not use the Wang et al. reference to definitively support the relative safety of a specific IV iron preparation.

6. While it is possible that pre- or concomitant medications may influence reactions to IV iron preparations, this has not been proven to date. The fact that these reactions are currently being reported to the FDA and other pharmacovigilance databases $[8,9]$ in the context of IV iron therapy at a level meeting established signal detection thresholds, and despite awareness of a potential role for pre- and concomitant medications following Drs. Auerbach's and 
Ballard's 2010 review [10], means that a clinical problem still exists. It cannot be assumed that this signal is solely attributable to pre- or concomitant medication. Validating and confirming the signal by appropriate studies investigating the mechanisms of reactions to IV iron preparations, e.g., complement activation-related pseudo-allergy [11], whether pre- or concomitant medications play a role, and how reactions to IV iron preparations can be effectively mitigated in clinical practice requires further research.

7. While true that the funding of the study was from the company that holds the marketing authorization for ferric carboxymaltose, this would also apply to the myriad of pharmaceutical company sponsored clinical trials, some of which have been quoted by Dr Auerbach in his letter. Thus, we do not consider this to be a valid criticism.

In conclusion, our manuscript used internationally accepted methods for analysis of the FAERS database [1] and therefore our findings contribute to the totality of evidence for the safety of IV iron products, along with evidence from randomized controlled trials and observational studies.

\section{Declarations}

Funding This study was supported by American Regent, USA, which holds the marketing rights for ferric carboxymaltose in the USA, and which provided funding to Advera Health Analytics, Inc. (Santa Rosa, California, USA) to conduct the FAERS data extraction and analysis.

Competing interests Henry Trumbo is a consultant for American Regent, Daiichi Sankyo, and Allergan. Lawrence T. Goodnough is a consultant for American Regent. Karolina Kaluza and Syed Numan are employees of American Regent, the manufacturers of ferric carboxymaltose.

Ethics approval Not applicable.

Consent to participate Not applicable.

Consent for publication Not applicable.

Availability of data and material (data transparency) The source data and analytics for this study were provided by Evidex ${ }^{\circledR}$, licensed by Advera Health Analytics, Inc., and are not publicly available. Healthcare Cost and Utilization Project (HCUP) data are made publicly available by the Agency for Healthcare Research and Quality (AHRQ).

Code availability (software application or custom code) Code used to conduct this analysis was provided by Evidex ${ }^{\circledR}$ (which is not publicly available), PostgreSQL (a free and open-source relational database), and Microsoft ${ }^{\circledR}$ Excel $^{\circledR}$.

Authors' contribution Henry Trumbo and Lawrence T. Goodnough contributed to the interpretation of data and to reviewing and writing of the original report. Karolina Kaluza and Syed Numan contributed to the conception and design of the analysis, the interpretation of data, and reviewing and writing of the original report. All authors contributed to the writing of this letter and read and approved the final version.
Open Access This article is licensed under a Creative Commons Attribution-NonCommercial 4.0 International License, which permits any non-commercial use, sharing, adaptation, distribution and reproduction in any medium or format, as long as you give appropriate credit to the original author(s) and the source, provide a link to the Creative Commons licence, and indicate if changes were made. The images or other third party material in this article are included in the article's Creative Commons licence, unless indicated otherwise in a credit line to the material. If material is not included in the article's Creative Commons licence and your intended use is not permitted by statutory regulation or exceeds the permitted use, you will need to obtain permission directly from the copyright holder. To view a copy of this licence, visit $\mathrm{http} / / /$ creativecommons.org/licenses/by-nc/4.0/.

\section{References}

1. Trumbo H, Kaluza K, Numan S, Goodnough LT. Frequency and associated costs of anaphylaxis- and hypersensitivity-related adverse events for intravenous iron products in the USA: an analysis using the US Food and Drug Administration Adverse Event Reporting System. Drug Saf. 2020. https://doi.org/10.1007/ s40264-020-01022-2.

2. Auerbach M. Comment on: "Frequency and Associated Costs of Anaphylaxis- and Hypersensitivity-Related Adverse Events for Intravenous Iron Products in the USA: An Analysis Using the US Food and Drug Administration Adverse Event Reporting System". Drug Saf. 2021. https://doi.org/10.1007/s40264-021-01058-y.

3. Wysowski DK, Swartz L, Borders-Hemphill BV, Goulding MR, Dormitzer C. Use of parenteral iron products and serious anaphylactic-type reactions. Am J Hematol. 2010;85(9):650-4. https:// doi.org/10.1002/ajh.21794.

4. Downing NS, Shah ND, Aminawung JA, et al. Postmarket safety events among novel therapeutics approved by the US Food and Drug Administration Between 2001 and 2010. JAMA. 2017;317(18):1854-63. https://doi.org/10.1001/jama.2017.5150.

5. Potts J, Genov G, Segec A, et al. Improving the safety of medicines in the European Union: from signals to action. Clin Pharmacol Ther. 2020;107(3):521-9. https://doi.org/10.1002/cpt.1678.

6. Achebe M, DeLoughery TG. Clinical data for intravenous iron-debunking the hype around hypersensitivity. Transfusion. 2020;60(6):1154-9. https://doi.org/10.1111/trf.15837.

7. Wang C, Graham DJ, Kane RC, et al. Comparative risk of anaphylactic reactions associated with intravenous iron products. JAMA. 2015;314(19):2062-8. https://doi.org/10.1001/jama.2015.15572.

8. Ehlken B, Nathell L, Gohlke A, et al. Evaluation of the reported rates of severe hypersensitivity reactions associated with ferric carboxymaltose and iron (III) isomaltoside 1000 in Europe based on data from eudravigilance and vigibase between 2014 and 2017. Drug Saf. 2019;42(3):463-71. https://doi.org/10.1007/ s40264-018-0769-5.

9. Nathell L, Gohlke A, Wohlfeil S. Reported severe hypersensitivity reactions after intravenous iron administration in the European Economic Area (EEA) before and after implementation of risk minimization measures. Drug Saf. 2020;43(1):35-43. https://doi. org/10.1007/s40264-019-00868-5.

10. Auerbach M, Ballard H. Clinical use of intravenous iron: administration, efficacy, and safety. Hematology. 2010;2010(1):338-47. https://doi.org/10.1182/asheducation-2010.1.338.

11. Szebeni J, Fishbane S, Hedenus M, et al. Hypersensitivity to intravenous iron: classification, terminology, mechanisms and management. Br J Pharmacol. 2015;172(21):5025-36. https://doi.org/10. 1111/bph.13268. 